\title{
Pseudohyperkalaemia in essential thrombocytosis: an important clinical reminder
}

\section{Correspondence} should be addressed to J Mizzi or C Rizzo or S Fava Email jean-marc.mizzi@gov.mt or christopher.rizzo@gov.mt or stephen.fava@gov.mt

\section{Summary}

An 82-year-old female was admitted to a general hospital due to progressive bilateral lower limb weakness. A T8-T9 extramedullary meningioma was diagnosed by MRI, and the patient was referred for excision of the tumour. During the patient's admission, she was noted to have persistent hyperkalaemia which was refractory to treatment. Following a review by an endocrinology team, a diagnosis of pseudohyperkalaemia secondary to thrombocytosis was made. This case demonstrates the importance of promptly identifying patients who are susceptible to pseudohyperkalaemia, in order to prevent its potentially serious consequences.

\section{Learning points}

- Pseudohyperkalaemia should be considered in patients with unexplained or asymptomatic hyperkalaemia. It should also be considered in those patients who are resistant to the classical treatment of hyperkalaemia.

- A diagnosis of pseudohyperkalaemia is considered when there is a difference of $>0.4 \mathrm{mmol} / \mathrm{L}$ of potassium between serum and plasma potassium in the absence of symptoms and ECG changes.

- In patients who are presenting with consistently elevated serum potassium levels, it may be beneficial to take venous blood gas and/ or plasma potassium levels to rule out pseudohyperkalaemia.

- Pseudohyperkalaemia may subject patients to iatrogenic hypokalaemia which can be potentially fatal.

- Pseudohyperkalaemia can occur secondary to thrombocytosis, red cell haemolysis due to improper blood letting techniques, leukaemia and lymphoma.

\section{Background}

Serum electrolytes are often taken as standard routine blood tests for almost all patients undergoing investigations, frequently daily in hospitalised patients. Pseudohyperkalaemia occurs when there is a raised serum potassium (1), with concomitant normal plasma potassium levels. A key understanding of pseudohyperkalaemia is essential for interpreting these investigations as true hyperkalaemia is potentially life-threatening and often presents insidiously.

Erroneously administering treatment for hyperkalaemia can predispose patients to iatrogenic complications of hypokalaemia such as ventricular fibrillation/tachycardia, paralytic ileus and respiratory depression (2).

\section{Case presentation}

An 82-year-old female presented to a general hospital emergency department with a 2-month history of progressive bilateral lower limb weakness; the patient was previously well and able to cope at home with ease but was now unable to bear weight. 
Her past medical history was significant for atrial fibrillation, essential thrombocytosis and tricuspid valve regurgitation.

The patient was on aspirin $75 \mathrm{mg}$ and hydroxyurea $500 \mathrm{mg}$ daily but admitted to not always being compliant. No potassium-sparing diuretics, angiotensin-converting enzyme inhibitors, angiotensin 2 receptor blockers and aldosterone antagonists were being administered and she was not taking potassium supplementation or herbal medications. Clinical examination revealed reduced power in both her lower limbs and a positive Babinski sign. An urgent spinal MRI showed a T8-T9 extramedullary meningioma compressing the spinal cord. The patient was admitted to neurosurgery and started on $1 \mathrm{~g}$ daily hydroxyurea after a haematology team review due to her persistent thrombocytosis $\left(900 \times 10^{9} / \mathrm{L}\left(140 \times 10^{\wedge} 9 / \mathrm{L}-400 \times 10^{\wedge} 9 / \mathrm{L}\right)\right)$.

She was initially treated for hyperkalaemia of 5.68 $\mathrm{mmol} / \mathrm{L}(3.5-5.1 \mathrm{mmol} / \mathrm{L})$ by the administration of $50 \mathrm{~mL}$ of 50\% dextrose with 10 units of fast acting insulin over $30 \mathrm{~min}$ and $10 \mathrm{~mL}$ of $10 \%$ calcium gluconate over $10 \mathrm{~min}$. Repeat testing of serum potassium showed potassium of $5.13 \mathrm{mmol} / \mathrm{L}$. Because of persistent slow atrial fibrillation, a temporary pacemaker was inserted prior to the neurosurgical procedure.

The patient underwent excision of the tumour and the haematology team advised to withhold hydroxyurea unless the patient's platelet count increases to above $700 \times 10^{9} / \mathrm{L}\left(140 \times 10^{9} / \mathrm{L}\right.$ to $\left.400 \times 10^{9} / \mathrm{L}\right)$.

During the recovery period, the patient's potassium was noted to be consistently elevated above $5.0 \mathrm{mmol} / \mathrm{L}$, which also coincided with an increase in the platelet count to levels over $1000 \times 10^{9} / \mathrm{L}$ and cessation of hydroxyurea

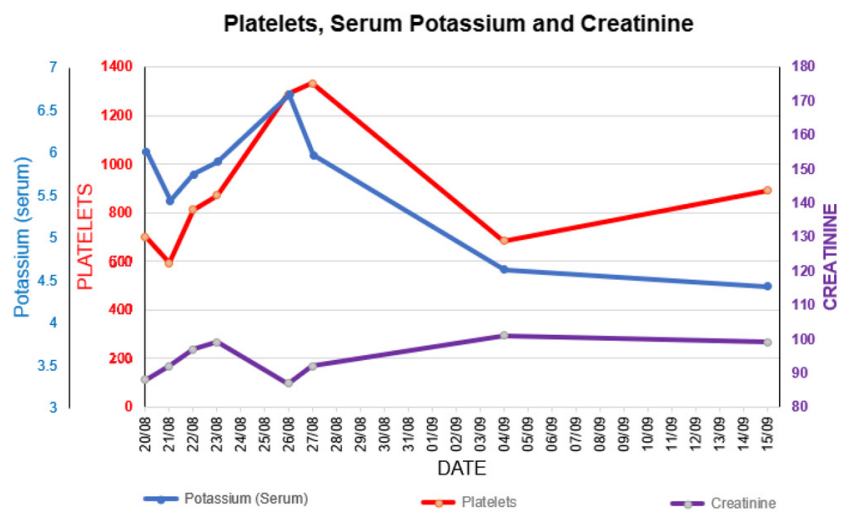

Figure 1

The correlation between platelets, serum potassium and creatinine.
(Fig. 1). On the first incidence of serum potassium raising to over $6.0 \mathrm{mmol} / \mathrm{L}$, the patient was again treated for hyperkalaemia according to the local hospital's clinical practice guidelines.

Following a review by the endocrinology team, pseudohyperkalaemia secondary to thrombocytosis was suspected and plasma potassium levels were requested. These came back as $3.7 \mathrm{mmol} / \mathrm{L}$ as opposed to 5.25 $\mathrm{mmol} / \mathrm{L}$ for serum potassium.

\section{Investigation}

One year prior to this admission, the patient had blood investigations done twice (serum potassium at 5.23 $\mathrm{mmol} / \mathrm{L}$ and $4.77 \mathrm{mmol} / \mathrm{L}$ and the patient had her platelet counts of $587 \times 10^{9} / \mathrm{L}$ and $627 \times 10^{9} / \mathrm{L}$ ).

During admission, the ECG showed slow atrial fibrillation but no changes associated with hyperkalaemia such as peaked $\mathrm{T}$ waves, shortened PR interval and ST segment depression. Serum creatinine was stable throughout her admission, with the highest-level reading recorded being $103 \mathrm{umol} / \mathrm{L}(44-80 \mathrm{umol} / \mathrm{L})$. $\mathrm{pH}$ values were also always within range.

Her serum potassium averaged $5.1 \mathrm{mmol} / \mathrm{L}$ (3.5-5.1 $\mathrm{mmol} / \mathrm{L})$. She had her serum electrolytes taking a total of 40 times. The patient had a total of 23 (57.5\%) readings above $5.0 \mathrm{mmol} / \mathrm{L}$, this is defined as hyperkalaemia. The maximum serum potassium recorded was $6.56 \mathrm{mmol} / \mathrm{L}$.

After a diagnosis of pseudohyperkalaemia was made, the average plasma potassium was $4.24 \mathrm{mmol} / \mathrm{L}$ (3.5-5.1 mmol/L).

A total of 40 complete blood counts were taken during the patient's admission. The average platelet count was $689 \times 10^{\wedge} 9 / \mathrm{L}\left(140 \times 10^{\wedge} 9 / \mathrm{L}-400 \times 10^{\wedge} 9 / \mathrm{L}\right)$. The patient had a total of 36 readings above $450 \times 10^{\wedge} 9 / \mathrm{L}$ which is defined as thrombocytosis (90\%). The maximum platelet count recorded was $1332 \times 10^{\wedge} 9 / \mathrm{L}$.

In-vitro haemolysis was effectively excluded as the patient's haemoglobin remained stable throughout her admission and was comparable to her levels prior to admission. The patient's haemoglobin ranged from 10.7 to $12.8 \mathrm{~g} / \mathrm{dL}$ (11.5-16.5 g/dL). Phosphate, uric acid and LDH levels were normal.

The tests requested by the endocrinology team included serum calcium, phosphate levels, urinary electrolytes, thyroid function tests, morning cortisol and renin-aldosterone levels and ratio. All these were noted to be within normal. 


\section{Outcome and follow-up}

Following the diagnosis of pseudohyperkalaemia secondary to thrombocytosis, plasma electrolytes were monitored and her potassium levels remained within normal levels. The patient had an uneventful postoperative recovery and was discharged to a rehabilitation hospital.

\section{Discussion}

Hyperkalaemia is a common clinical condition if left untreated can be potentially life-threatening. It occurs due to the imbalance between intake and renal excretion or from a maldistribution between the intracellular and extracellular compartments. The symptoms of hyperkalaemia are often insidious but due to the widespread use of electrolyte testing in inpatients, it is often encountered in clinical practice.(3)Hyperkalaemia may present with nausea, palpitations, muscle pain or paraesthesia and it may show ECG changes such as peaked T-waves, QRS widening and ST-segment depression. These ECG changes are non-specific and non-sensitive, and there is no evidence that it should influence treatment intensity or monitoring. $(4,5)$

Treatment of hyperkalaemia in the acute setting involves shifting extracellular potassium intracellularly. Guidelines for hyperkalaemia management can vary but they normally utilise fast-acting insulin with dextrose infusion (to prevent hypoglycaemia) and the use of nebulised or i.v. beta- ${ }_{2}$ agonists. Exchange resins are often employed in the management of hyperkalaemia, they were not used by the attending physician in this case. Calcium gluconate is often added due to its cardioprotective effects. Most hyperkalaemia guidelines also suggest ECG monitoring and the utilisation of arterial/venous blood gases. Patients with severe hyperkalaemia are also treated with renal replacement therapy if renal function is poor or the patient is not responding to treatment. Erroneously treating pseudohyperkalaemia, as was the case in this patient, may precipitate hypokalaemia which may present with muscle weakness, myalgia, tremor, ascending paralysis, intestinal paralysis, respiratory failure, cardiac failure and arrhythmias.(2)This case demonstrates that clinicians must maintain a high degree of clinical suspicion, especially in patients with thrombocytosis, in order to avoid iatrogenic hypokalaemia.

Pseudohyperkalaemia is an in vitro phenomenon that was first described in 1955 by Hartmann and Mellinkoff (6) and occurs when there is elevated serum potassium in a specimen with concomitant normal plasma potassium and endogenous blood levels (4). It was suggested by Singh et al that there must be a difference of $0.4 \mathrm{mmol} / \mathrm{L}$ between plasma and serum potassium levels for pseudohyperkalaemia to be considered. (7) Pseudohyperkalaemia is also characterised by the absence of ECG changes and symptoms of hyperkalaemia together with a lack of response to the classical treatment of hyperkalaemia. Furthermore, it has been demonstrated that patients with pseudohyperkalaemia secondary to thrombocytosis occur at platelet concentrations above $600 \times 10^{9} / \mathrm{L}(8)$. Figure 1 shows that hyperkalaemia occurred when measuring potassium in platelet concentrations $>600 \times 10^{9} / \mathrm{L}$, even in our limited data set in this single patient.

Pseudohyperkalaemia has several potential causes ( refer to Table 1). One of the most common is haemolysis of red blood cells during prolonged or improper blood collection. This often occurs when a patient has difficult venous access. Other causes include leucocytosis, thrombocytosis and improper storage of blood samples. In leucocytosis increased cellular fragility leading to increased amounts of intracellular potassium being released into the circulation is thought to be the underlying cause. (9)

Blood for the measurement of serum potassium is normally taken in bottles that contain silica and an acrylic gel polymer. The silica within these blood sample bottles promotes coagulation whereas the acrylic gel polymer stabilises the sample and enables its analysis. Platelet aggregates degranulate in these blood samples and release intracellular potassium. (8) In thrombocytosis, there are more platelets that release more intracellular potassium. Plasma electrolytes are taken in a specialised lithium heparinised bottle where tube inversions ensure mixing of anticoagulant (heparin) with blood to prevent clotting. (1) Prevention of clotting, therefore, prevents the release of intracellular potassium from platelets. The increase in serum potassium, from platelets, initially shows a linear increase at higher platelet concentrations in thrombocytosis. This is then followed by a drop

Table 1 Causes of pseudohyperkalaemia (the serum potassium exceeds the plasma potassium by more than 0.4 $\mathrm{mmol} / \mathrm{L})$

- Prolonged tourniquet application prior to venepuncture

- Haemolysis during venepuncture

- Samples undergoing centrifugation prior to clot formation

- Leukocytosis (white cell count greater than $>70$ 000/mm³)

- Thrombocytosis

- Familial pseudohyperkalaemia

- Hereditary spherocytosis 
(hypothesised to be due to the re-entry of potassium into the red blood cell as homeostasis), making the trend not truly linear. (10)

In conclusion, this case serves as an important clinical reminder that pseudohyperkalaemia can occur in patients with thrombocytosis. Being aware of this phenomenon could avoid inadvertent iatrogenically induced hypokalaemia.

\section{Declaration of interest}

The authors declare that there is no conflict of interest that could be perceived as prejudicing the impartiality of the research reported.

\section{Funding}

This research did not receive any specific grant from any funding agency in the public, commercial or not-for-profit sector.

\section{Patient consent}

Written informed consent for publication of the clinical details was obtained from the patient.

\section{Author contribution statement}

All the authors were directly involved in the clinical work-up of the patient. J M M edited the paper. C R supervised the clinical follow-up of the patients and revised the final version of the manuscript

\section{References}

1 Van Elslande J, Dominicus T, Toelen J, Frans G \& Vermeersch P. A case of severe pseudohyperkalaemia due to muscle contraction. Biochemia Medica 202030 021004. (https://doi.org/10.11613/ BM.2020.021004)

2 Šálek T. Pseudohyperkalemia - potassium released from cells due to clotting and centrifugation - a case report. Biochemia Medica 201828 011002. (https://doi.org/10.11613/BM.2018.011002)

3 Lehnhardt A \& Kemper MJ. Pathogenesis, diagnosis and management of hyperkalemia. Pediatric Nephrology 201126 377-384. (https://doi.org/10.1007/s00467-010-1699-3)

4 Kardalas E, Paschou SA, Anagnostis P, Muscogiuri G, Siasos G \& Vryonidou A. Hypokalemia: a clinical update. Endocrine Connections 20187 R135-R146. (https://doi.org/10.1530/EC-18-0109)

5 Montague BT, Ouellette JR \& Buller GK. Retrospective review of the frequency of ECG changes in hyperkalemia. Clinical Journal of the American Society of Nephrology 20083 324-330. (https://doi. org/10.2215/CJN.04611007)

6 Hartmann RC. The relationship of platelets to the serum potassium concentration. Journal of Clinical Investigation 195534938.

7 Singh PJ, Zawada ET \& Santella RN. A case of 'reverse' pseudohyperkalemia. Mineral and Electrolyte Metabolism 199723 58-61.

8 Alizadeh K, Hadjinicolaou AV, Hadjittofi C \& Shankar A. Postsplenectomy thrombocytosis with pseudohyperkalaemia. BMJ Case Reports 20152015 bcr2015211720. (https://doi.org/10.1136/ bcr-2015-211720)

9 Ahmed R \& Isaac AM. Postsplenectomy thrombocytosis and pseudohyperkalemia in trauma: A case report and review of literature. Journal of Trauma 200967 E17-E19. (https://doi. org/10.1097/01.ta.0000238653.55029.cd)

10 Asirvatham JR, Moses V \& Bjornson L. Errors in potassium measurement: a laboratory perspective for the clinician. North American Journal of Medical Sciences 20135 255-259. (https://doi. org/10.4103/1947-2714.110426)

Received in final form 9 March 2021 Accepted 12 April 2021 